Supplementary Material for

\title{
Accurate Estimation of Solvent Accessible Surface Area for Coarse- Grained Biomolecular Structures with Deep Learning
}

Tiejun Dong1,2,3,4, Tong Gong ${ }^{4}$ and Wenfei $\mathrm{Li}^{1,2,3^{*}}$

${ }^{1}$ National Laboratory of Solid State Microstructure, Department of Physics, and Collaborative Innovation Center of Advanced Microstructures, Nanjing University, Nanjing 210093, China; ${ }^{2}$ Wenzhou Institute, University of Chinese Academy of Sciences,

Wenzhou, Zhejiang 325001, China; ${ }^{3}$ Oujiang Laboratory, Wenzhou, Zhejiang 325000, China; ${ }^{4}$ Institute of Drug R\&D, Nanjing University, Nanjing 210093, China. 


\section{S.1 DeepCGSA performance with different hyperparameters.}

To investigate the sensitivity of the DeepCGSA performance to the network hyperparameters, we constructed the network architectures with different depth and sizes for the protein structures (Supplementary Table S3) and RNA structures (Supplementary Table S4). We found that the network with less than 10 ResNet blocks could provide sufficient performance. Further increasing the depth and size of the network only gives a slightly better performance. Therefore, we choose to use a network architecture with moderate size in the final applications (Supplementary Table S2). In the DeepCGSA network, the output of the 1D-ResNet blocks is one part of the input for the subsequent 2D-ResNet blocks. Therefore, the data structure of the 1D-ResNet output needs to be reshaped in order to match the 2D-ResNet input structure. We introduced two operations to match the data structure, including A) matrix duplication and B) transposition of the duplicated matrix. The combinations of the above two operations were used to reshape the data structure (Supplementary Figure S4). We also tested the performance of the DeepCGSA network by removing one of the operations, and the results showed that the combination of the two operations gives better performance on the residue-wise SASA estimations (Supplementary Table S5). In addition, in order to map the 2D-ResNet output to the final SASA values, we applied "mean" or "sum" operations to reduce the dimensionality of the output data. We found that with the "sum" operation, the DeepCGSA can achieve a smaller loss and better correlation (Supplementary Table S6).

\section{S.2 Details of network training}

The reference SASA values used for training and testing were calculated by using NACCESS based on the all-atom structures in the data sets, which were considered as accurate values. ${ }^{1}$ The SASA values at the CG bead level were used in the training. For each structure in the training set, the one-hot encoding of the sequence information and the distance matrix of the CG beads were prepared and fed into the input of the DeepCGSA network. For each structure in the training set, the one-hot encoding of the sequence information and the distance matrix of the CG beads were prepared and fed into the input of the DeepCGSA network. The 99th percentile of the distance and SASA values in training set were used to normalize the data. We used the Adam optimizer to train the DeepCGSA model with the Mean Square Error (MSE) being used as the loss function. ${ }^{2}$ The batch size of each iteration was set as 4 and the structures in training set were randomly shuffled before training. The network training was performed by two stages, including the model training stage and the model refining stage. The learning rate during the training stage was set as $3.0 \times 10^{-4}$, and it decayed exponentially by a rate of 0.99 with the iterations. The training terminated after the training loss got stabilized. The output of the model was activated using the following custom function:

$$
f(x)=\left\{\begin{aligned}
-\log (1-x), & x<0 \\
x, & x \geq 0
\end{aligned}\right.
$$

In the model refining stage, the model was first trained by 5000 iterations with the learning rate of $1 \times 10^{-6}$. Then the activation function was changed to ReLU and the model was trained for another 5000 iterations. The MSE values were recorded for each iteration during the training, and the final converged MSE value was used to characterize the quality of training process.

\section{S.3 Estimation of nonpolar solvation free energies}

In the implicit solvent models used in the molecular simulations, the solvation free energy can be decomposed into polar part and non-polar part, i.e., $\Delta G_{\text {solv }}=$

$\Delta G_{\text {pol,solv }}+\Delta G_{\mathrm{np}, \text { solv }}{ }^{3}$ The polar part solvation free energy $\Delta G_{\text {pol,solv }}$ is often calculated based on Poisson-Boltzmann equation, Generalized-Born solvation model, Debye-Hückel theory, or other continuum solvation models. ${ }^{4}$ The non-polar solvation free energy can be calculated based on the SASA values by $\Delta G_{\mathrm{np}, \mathrm{solv}}=\gamma \cdot \mathrm{SASA}+b$, where $\gamma$ is the surface tension constant and $b$ is a correction constant. ${ }^{5}$ The values of these parameters $\left(\gamma=0.005 \mathrm{kcal} \cdot \mathrm{mol}^{-1} \cdot \AA^{-2}\right.$ and $\mathrm{b}$ $=0.860 \mathrm{kcal} \cdot \mathrm{mol}^{-1}$ ) were taken from Ref.5. 


\section{S.4 Supplementary figures and legends.}

A

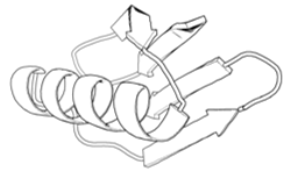

B

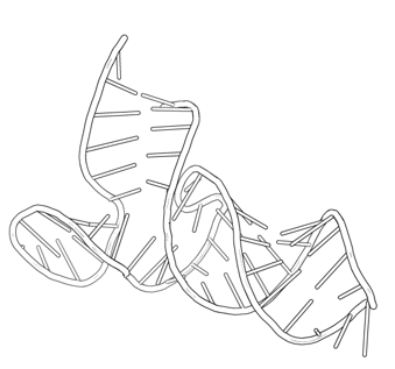

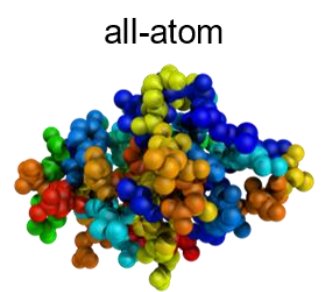

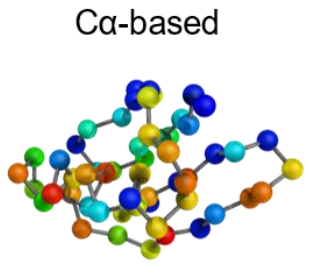

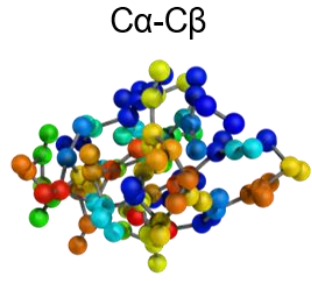
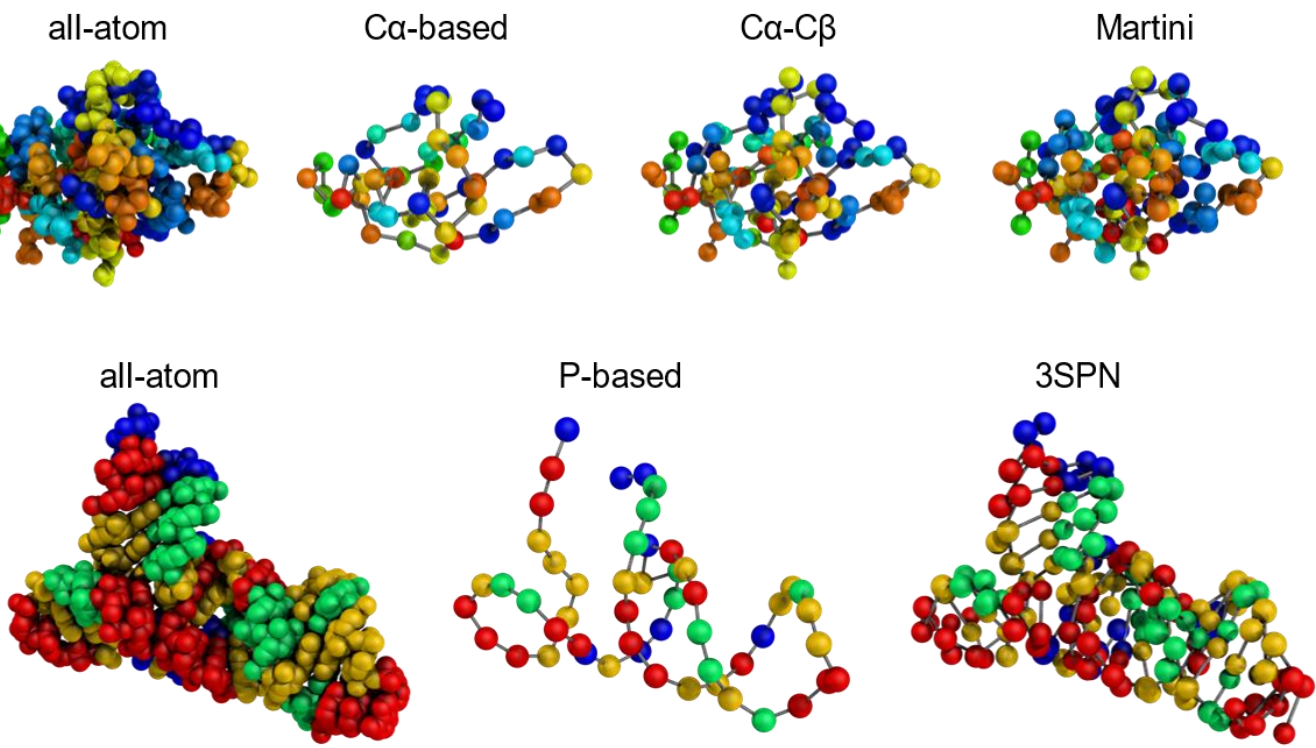

Supplementary Figure S1. Illustration of structure mapping from all-atom model to different coarse-grained models for protein G (PDB code: 1PGB). (A) and tRNA (PDB code: 3E5E) (B). The cartoon structures were also shown (left). In the all-atom model, each sphere represents one heavy atom.

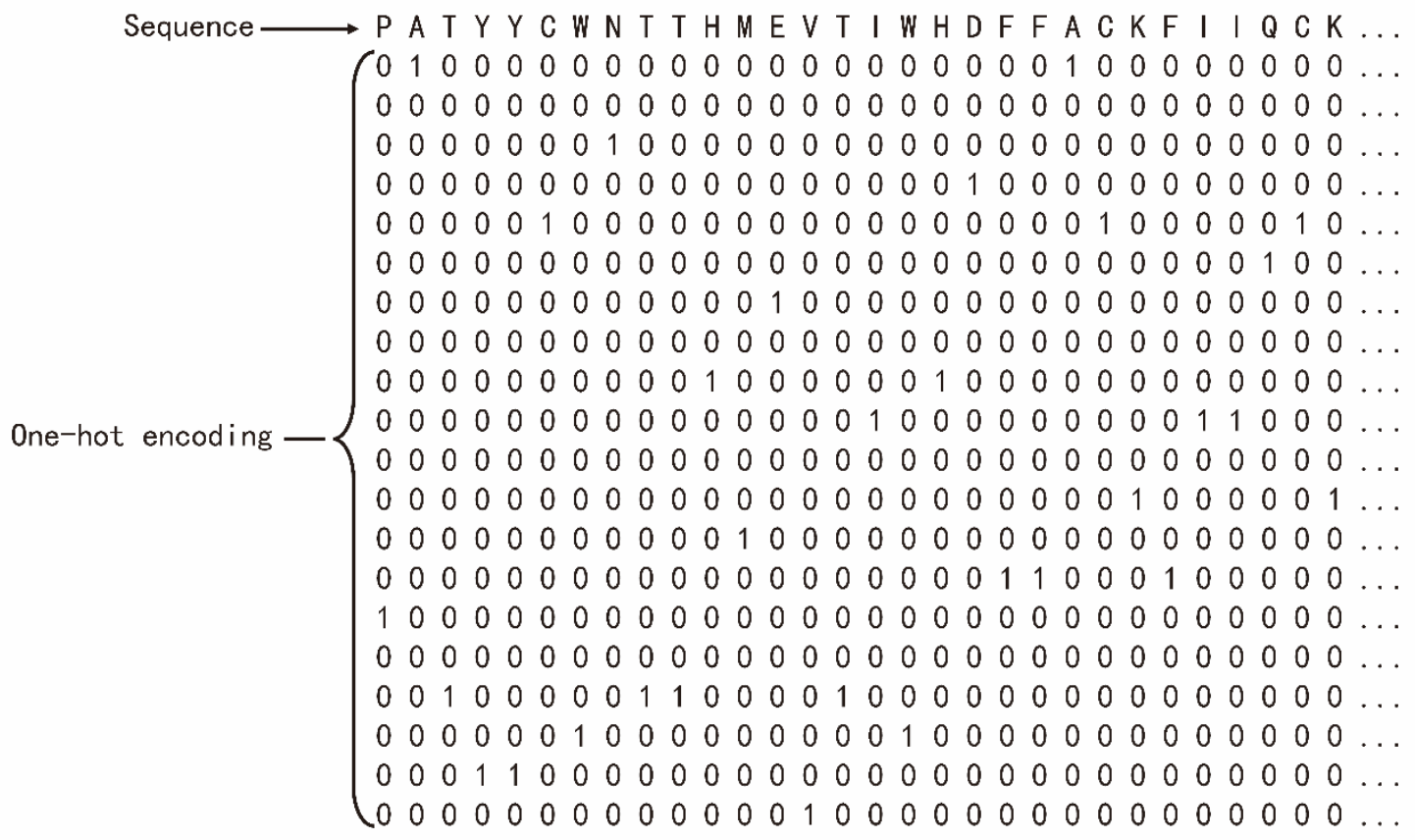

Supplementary Figure S2. Illustration of one-hot encoding of a protein sequence. 

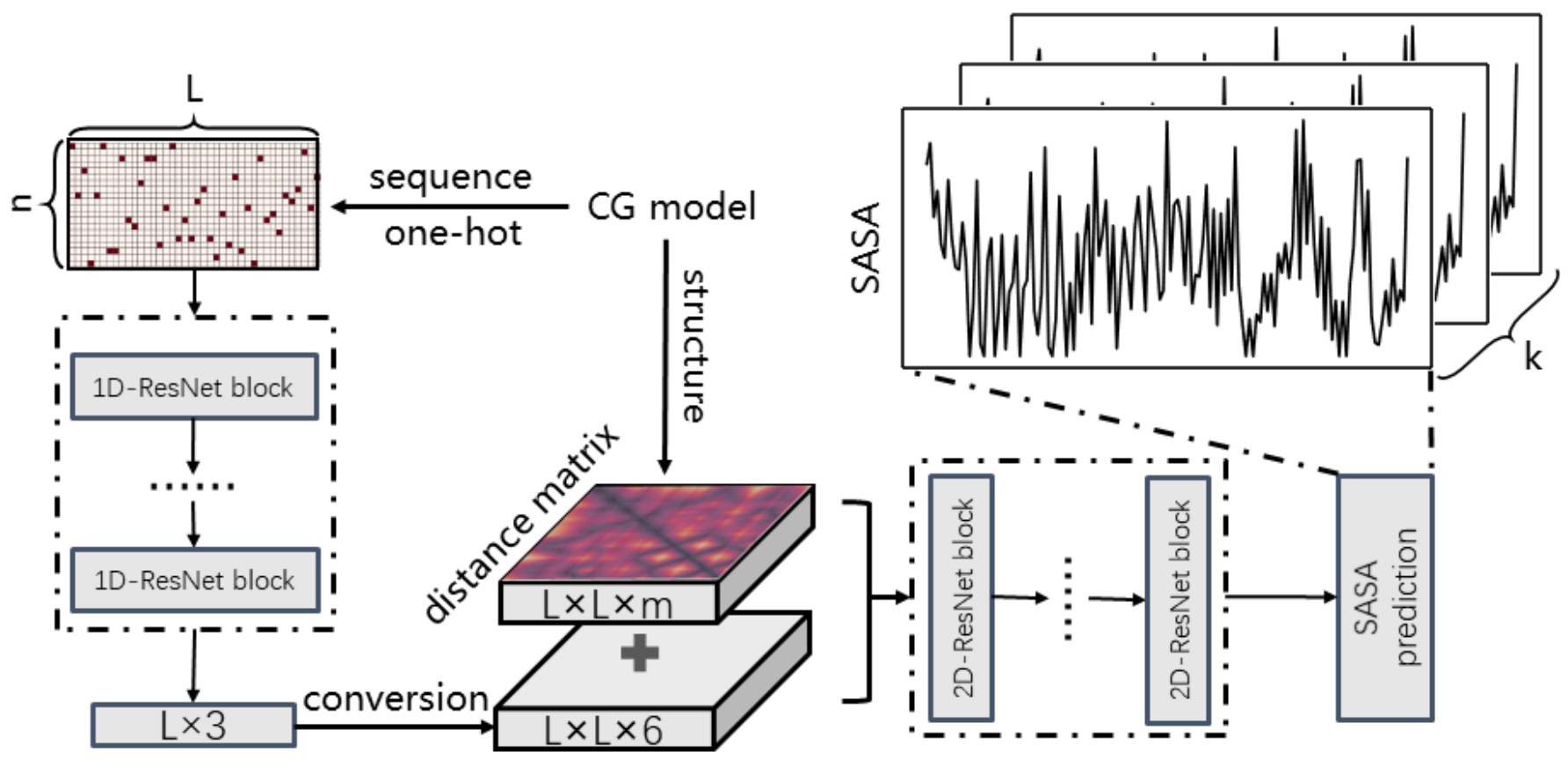

Supplementary Figure S3. Schematic diagram of the DeepCGSA architecture, L, m, n and k were explained in Methods.

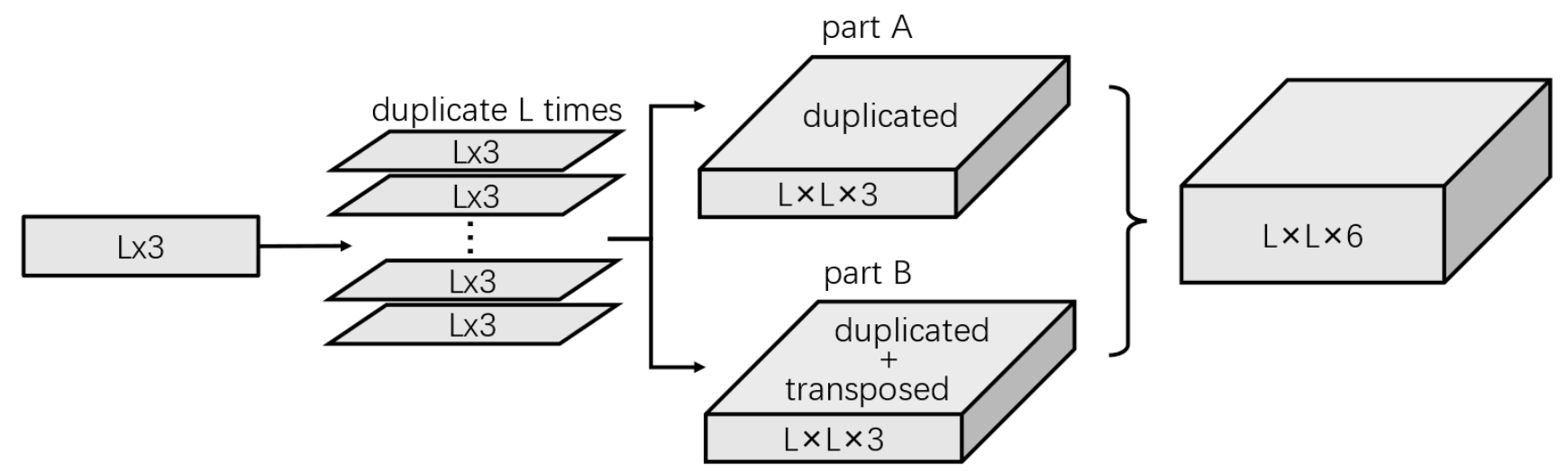

Supplementary Figure S4. Schematic diagram for the data structure reshaping from 1D-ResNet output with L×3 tensor structure to $2 \mathrm{D}-\mathrm{ResNet}$ input with $\mathrm{L} \times \mathrm{L} \times 6$ tensor structure by combination of duplication (part A) and transposition of the duplicated tensor (part B) operations. L was explained in Methods. 


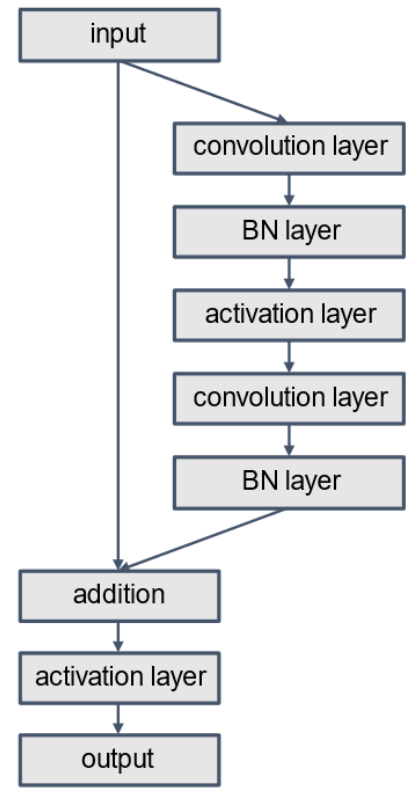

Supplementary Figure S5. Schematic diagram of a ResNet block architecture. BN: batch normalization.
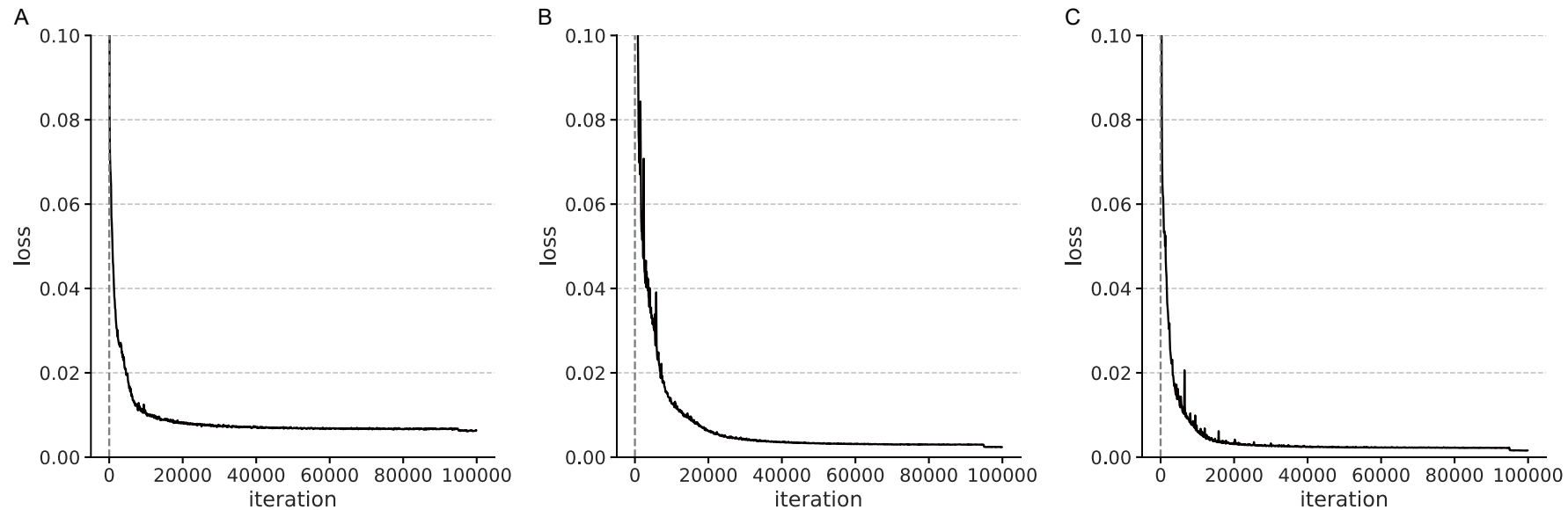

Supplementary Figure S6. Training loss of DeepCGSA as a function of iteration steps for C $\alpha$-based structures (A), C $\alpha$-C $\beta$ structures (B) and Martini structures $(\mathrm{C})$. 
A
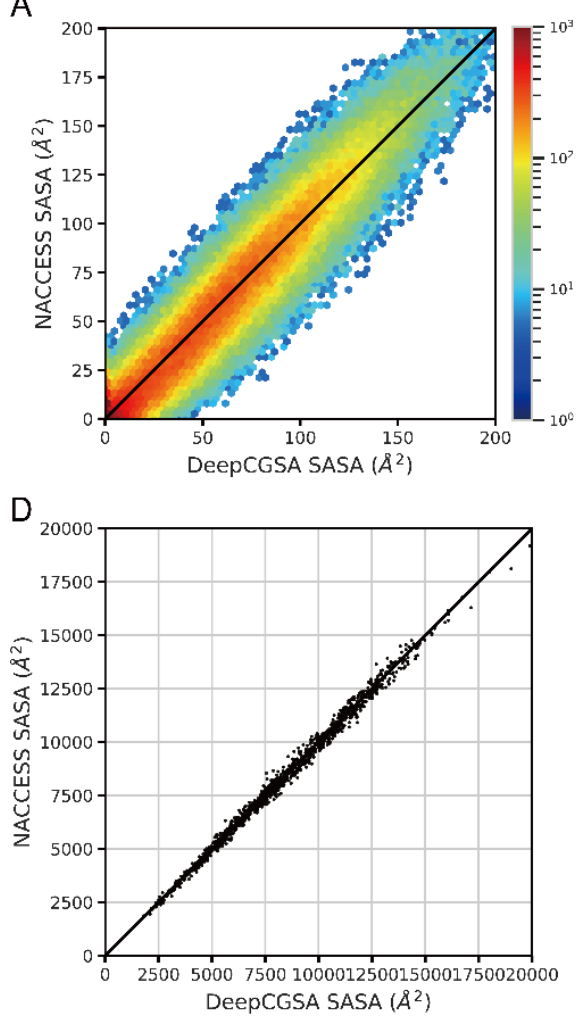

B

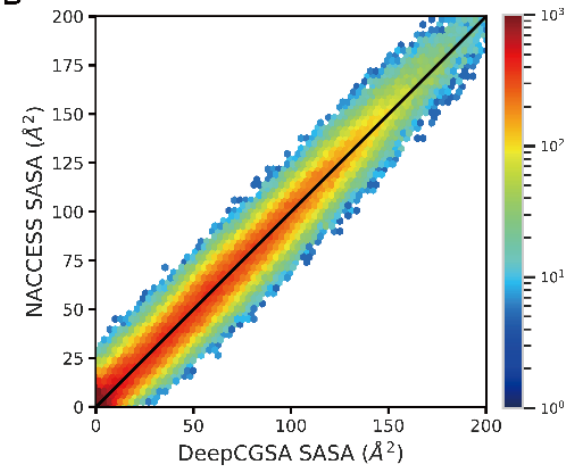

E

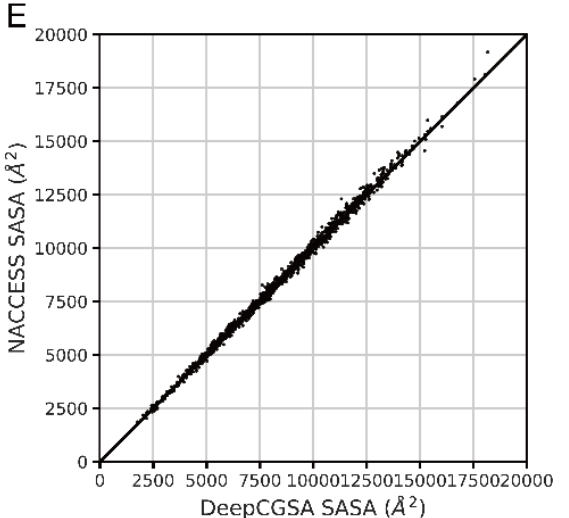

C

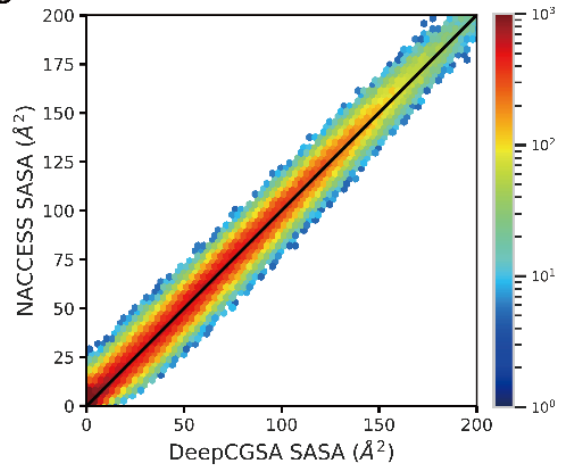

$\mathrm{F}$

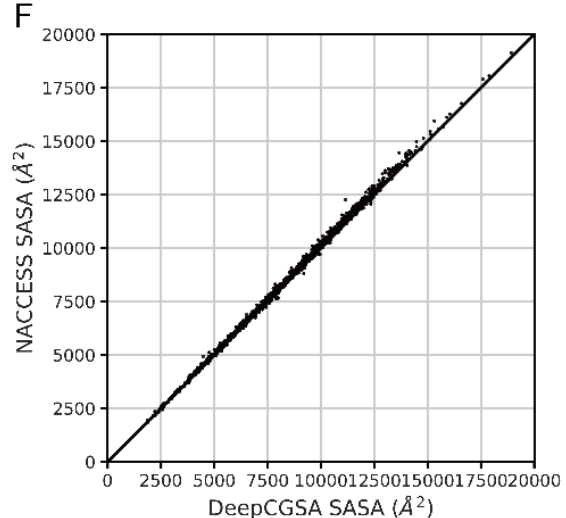

Supplementary Figure S7. Correlation plots between the SASA values estimated by DeepCGSA with coarse-grained protein structures and the reference values calculated by NACCESS with all-atom structures. (A-C) Residue-wise SASA estimation for C $\alpha$-based structures (A), C $\alpha$-C $\beta$ structures (B) and Martini structures (C) in all test set. (D-F) Protein-wise SASA estimation for $\mathrm{C} \alpha$-based structures (D), $\mathrm{C} \alpha-\mathrm{C} \beta$ structures (E) and Martini structures (F) in all test set.

A

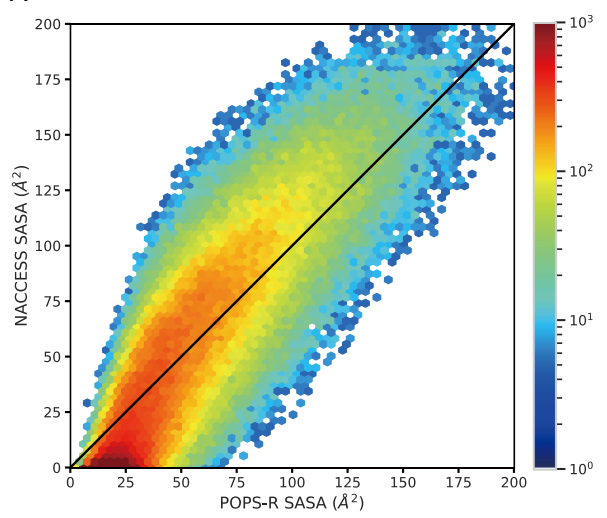

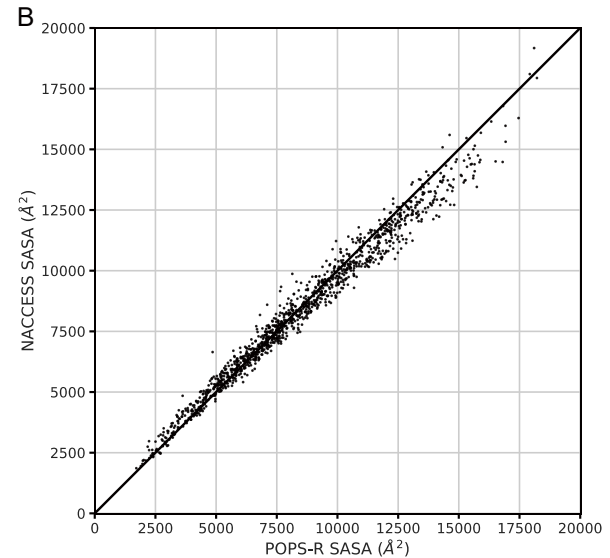

Supplementary Figure S8. Correlation plots between the SASA values estimated by POPS-R with C $\alpha$-based protein structures and the reference values by NACCESS with all-atom structures at the residue-wise level (A) and protein-wise level (B) for all the proteins in the test set. 


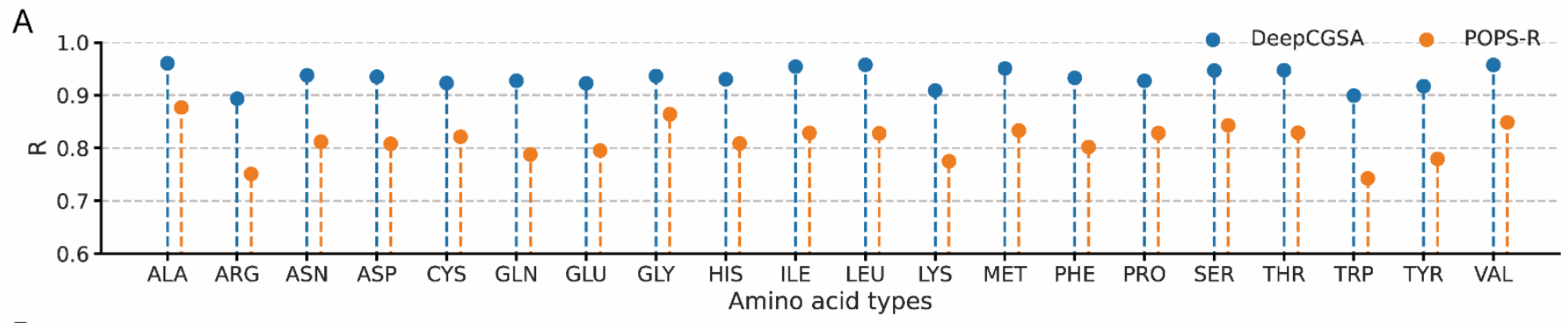

B
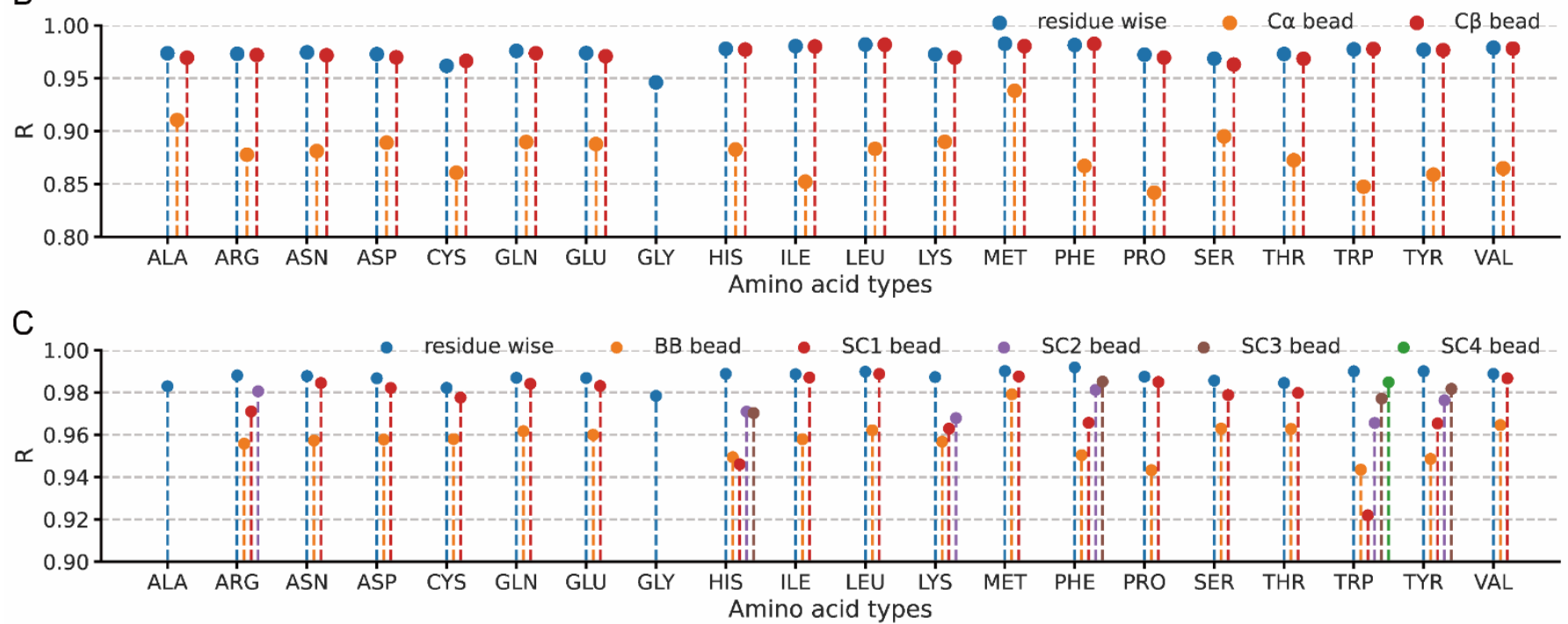

Supplementary Figure S9. Amino acid type dependence of DeepCGSA performance. (A) Pearson correlation coefficients between the estimated SASA values and the reference values for the DeepCGSA and POPS-R methods using the $\mathrm{C} \alpha$-based CG structures for the proteins in the test set. The results for residuewise SASA were shown. (B) Same as (A) but using the C $\alpha-C \beta$ structures. The results for residue-wise SASA, C $\alpha$-bead SASA, and the side-chain bead SASA were shown. (C) Same as (A) but using the Martini structures. The results for residue-wise SASA and Martini-bead SASA were shown. 

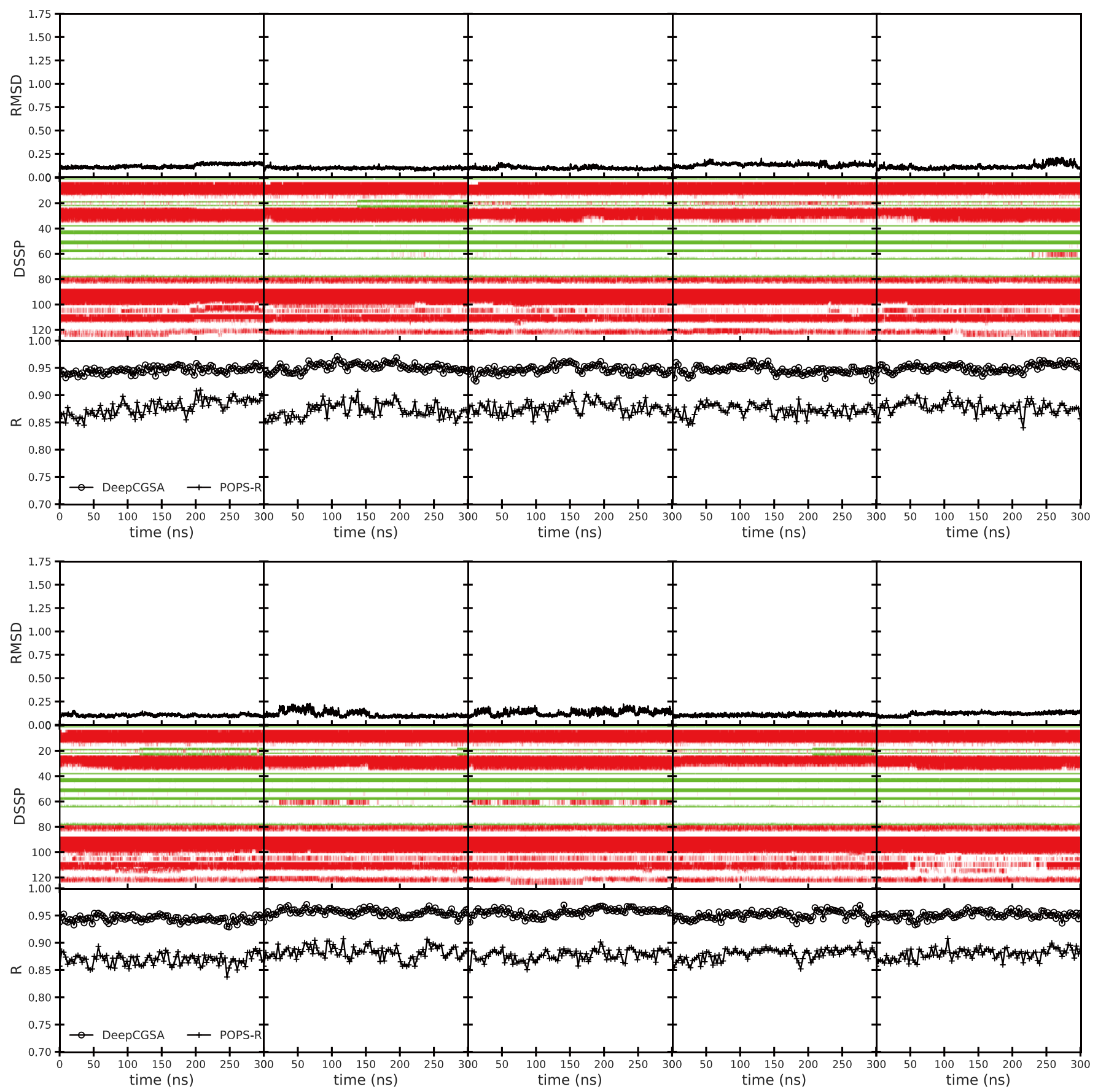

Supplementary Figure S10. Root mean square deviations (RMSD), secondary structure contents (DSSP), and correlation coefficients between estimated SASA values by DeepCGSA/POPS-R and the reference values for lysozyme structures sampled from MD simulations at $300 \mathrm{~K}$. Results for 10 independent MD simulations with the lengths of $300 \mathrm{~ns}$ were shown. 

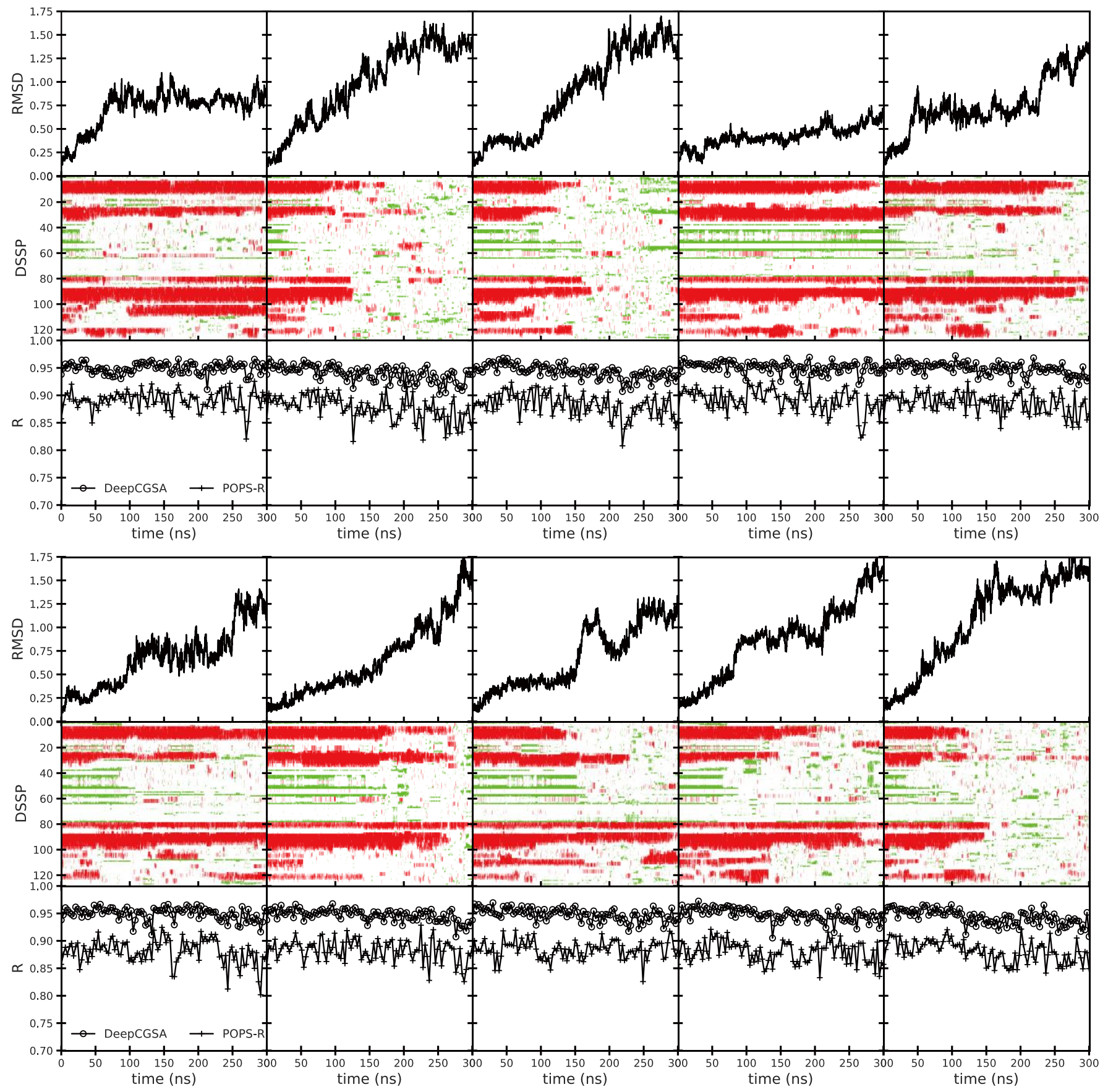

Supplementary Figure S11. Root mean square deviations (RMSD), secondary structure contents (DSSP), and correlation coefficients between estimated SASA values by DeepCGSA/POPS-R and the reference values for lysozyme structures sampled from MD simulations at $500 \mathrm{~K}$. Results for 10 independent MD simulations with the lengths of 300ns were shown. 

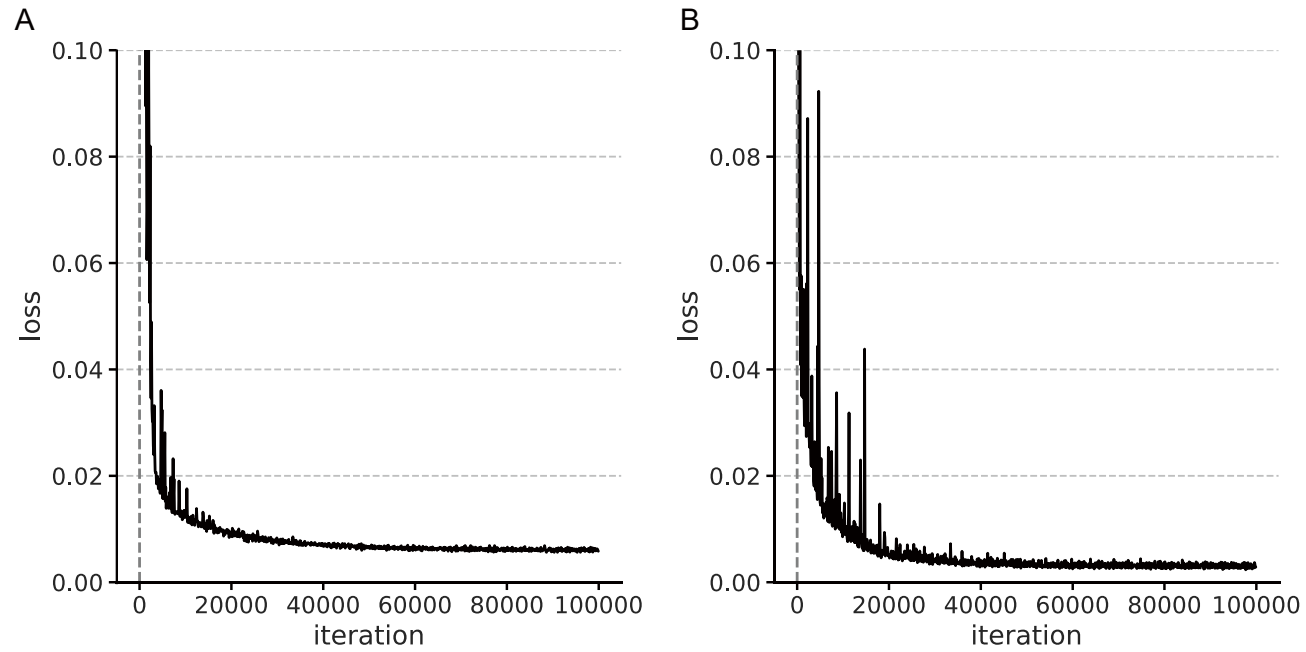

Supplementary Figure S12. Training loss of DeepCGSA as a function of iteration steps for P-based structures (A) and 3SPN structures (B).

A

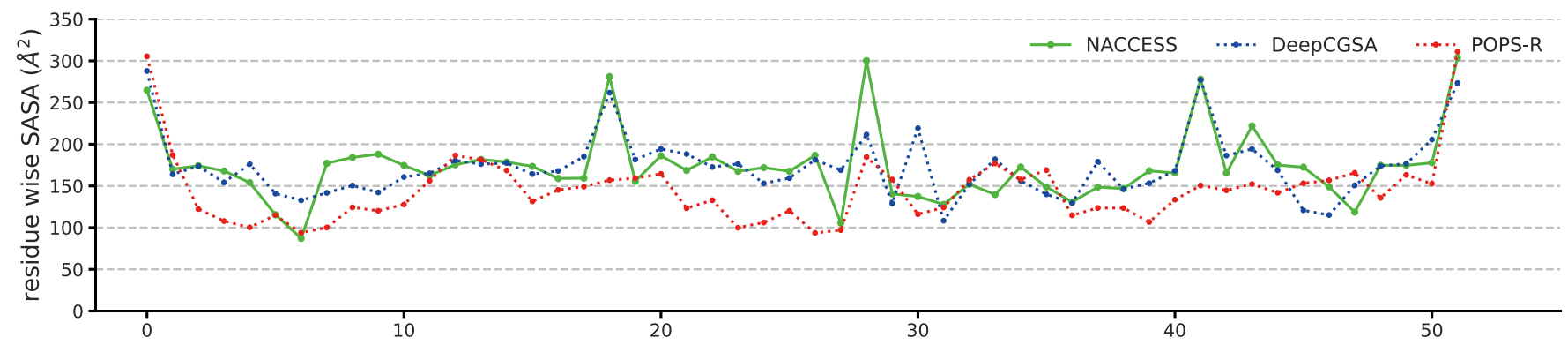

B

sequence

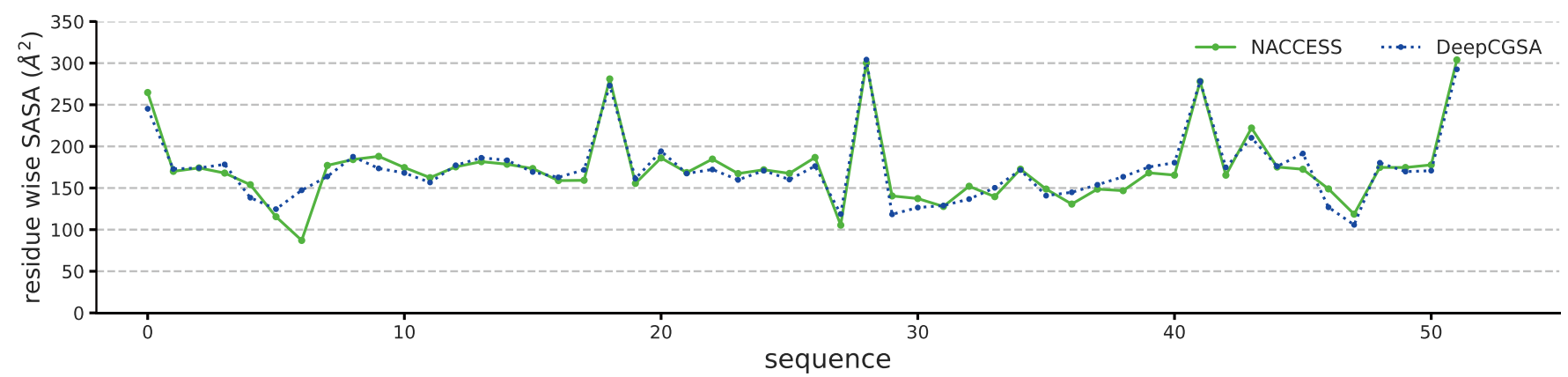

Supplementary Figure S13. Estimating Residue-wise SASA of a representative tRNA (PDB code: 3E5E). (A) Residue-wise SASA values estimated by DeepCGSA (blue) using P-based structure of tRNA. For comparison, the reference values (green) and the results by POPS-R (red) were also shown. (B) Residuewise SASA values estimated by DeepCGSA (blue) and the reference SASA values (green) using 3SPN of tRNA. 

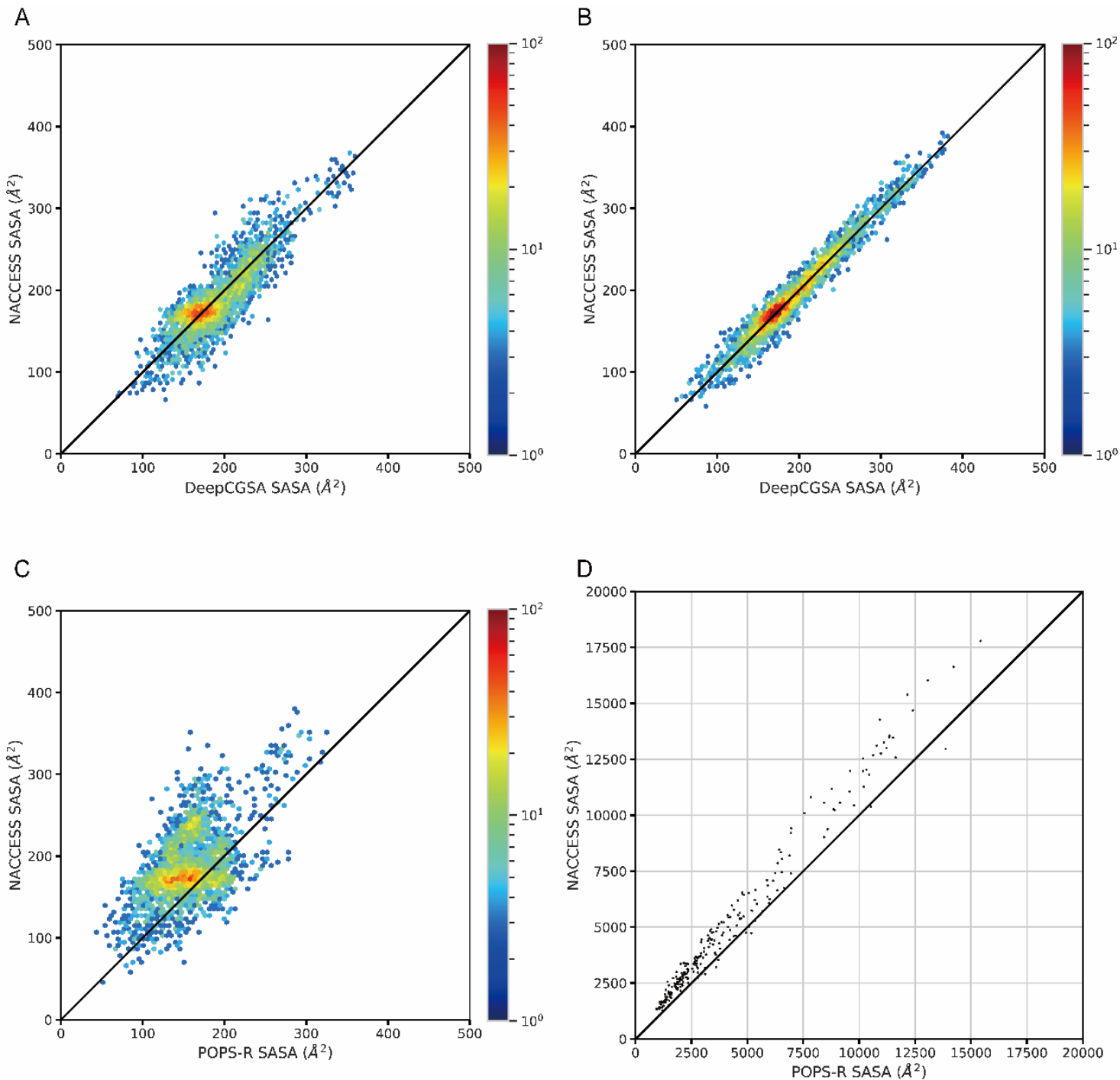

Supplementary Figure S14. Correlation plots between the estimated SASA values and reference values. (A, B) Residue-wise correlation plots between the estimated SASA values by DeepCGSA and the reference SASA values for P-based structures (A) and 3SPN structures (B) based on all the RNA structures in the test set. (C, D) Correlation plots between the SASA values estimated by POPS-R with P-based RNA structures and the reference values by NACCESS with allatom structures at the residue-wise level (A) and RNA-wise level (B) for all the RNAs in the test set. 
A

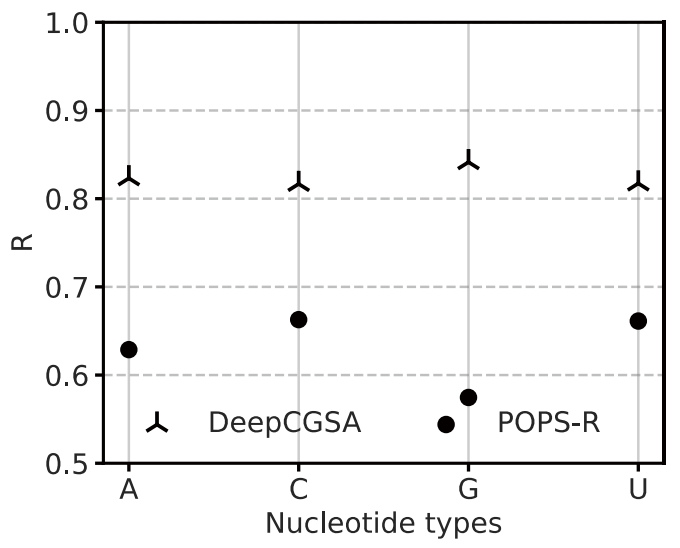

B

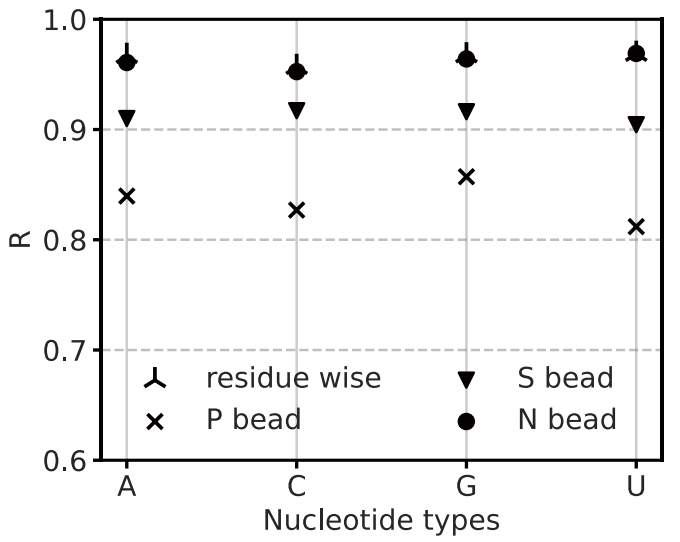

Supplementary Figure S15. Nucleotide type dependence of DeepCGSA performance. (A) Pearson correlation coefficients R between the estimated SASA values and the reference values for the DeepCGSA and POPS-R methods using the P-based CG structures for the RNAs in the test set. (B) Same as (A) but using the 3SPN structures. The results for residue-wise SASA, P bead SASA, S bead SASA and N bead SASA were shown. 


\section{S.5 Supplementary tables.}

Supplementary Table S1. Number of samples in training set, validation set and test set.

\begin{tabular}{lllll}
\hline CG type & training set & validation set & test set & Max. length \\
\hline C $\alpha$-based & 9916 & 1215 & 1252 & 300 \\
C $\alpha$-C $\beta$ & 9937 & 1222 & 1255 & 300 \\
Martini & 9938 & 1222 & 1256 & 300 \\
P-based & 1777 & 287 & 205 & 558 \\
3SPN & 1777 & 287 & 265 & 558 \\
\hline
\end{tabular}

Supplementary Table S2. Hyperparameters of DeepCGSA for different CG models of the proteins and RNAs.

\begin{tabular}{|c|c|c|}
\hline CG type & 1D-ResNet & 2D-ResNet \\
\hline C $\alpha$-based & \multirow{3}{*}{$\begin{array}{l}\text { - } 1 \text { convolution layer (with BN and activation layer) with } 4 \text { filters } \\
\text { and } 20 \text { kernel size; } \\
\text { - } 1 \text { convolution layer (with BN and activation layer) with } 8 \text { filters } \\
\text { and } 15 \text { kernel size; } \\
\text { - } 11 \text { D-ResNet block with } 8 \text { filters and } 15 \text { kernel size; } \\
\text { - } 2 \text { 1D-ResNet blocks with } 16 \text { filters and } 15 \text { kernel size; } \\
\text { - } 1 \text { convolution layer (with BN and activation layer) with } 3 \text { filters } \\
\text { and } 1 \text { kernel size; }\end{array}$} & $\begin{array}{l}\text { - } 2 \text { 2D-ResNet blocks with } 8 \text { filters and } 5 \times 5 \text { kernel size; } \\
\text { - } 3 \text { 2D-ResNet blocks with } 16 \text { filters and } 5 \times 5 \text { kernel size; } \\
\text { - } 1 \text { convolution layer with } 1 \text { filter and } 1 \times 1 \text { kernel size; }\end{array}$ \\
\hline $\mathrm{C} \alpha-C \beta$ & & $\begin{array}{l}\text { - } 2 \text { 2D-ResNet blocks with } 8 \text { filters and } 5 \times 5 \text { kernel size; } \\
\text { - } 3 \text { 2D-ResNet blocks with } 16 \text { filters and } 5 \times 5 \text { kernel size; } \\
\text { - } 1 \text { convolution layer with } 2 \text { filters and } 1 \times 1 \text { kernel size; }\end{array}$ \\
\hline Martini & & $\begin{array}{l}\text { - } 2 \text { 2D-ResNet blocks with } 8 \text { filters and } 5 \times 5 \text { kernel size; } \\
\text { - } 3 \text { 2D-ResNet blocks with } 16 \text { filters and } 5 \times 5 \text { kernel size; } \\
\text { - } 1 \text { convolution layer with } 1 \text { filter and } 1 \times 1 \text { kernel size; }\end{array}$ \\
\hline P-based & \multirow{2}{*}{$\begin{array}{l}\text { - } 1 \text { convolution layer (with BN and activation layer) with } 4 \text { filters } \\
\text { and } 5 \text { kernel size; } \\
\text { - } 1 \text { 1D-ResNet block with } 8 \text { filters and } 5 \text { kernel size; } \\
\text { - } 21 \text { 1D-ResNet blocks with } 16 \text { filters and } 5 \text { kernel size; } \\
\text { - } 1 \text { convolution layer (with BN and activation layer) with } 3 \text { filters } \\
\text { and } 1 \text { kernel size; }\end{array}$} & $\begin{array}{l}\text { - } 1 \text { 2D-ResNet blocks with } 8 \text { filters and } 5 \times 5 \text { kernel size; } \\
\text { - } 2 \text { 2D-ResNet blocks with } 16 \text { filters and } 5 \times 5 \text { kernel size; } \\
\text { - } 1 \text { convolution layer with } 1 \text { filter and } 1 \times 1 \text { kernel size; }\end{array}$ \\
\hline 3SPN & & $\begin{array}{l}\text { - } 2 \text { 2D-ResNet blocks with } 12 \text { filters and } 5 \times 5 \text { kernel size; } \\
\text { - } 3 \text { 2D-ResNet blocks with } 16 \text { filters and } 5 \times 5 \text { kernel size; } \\
\text { - } 1 \text { convolution layer with } 3 \text { filter and } 1 \times 1 \text { kernel size; }\end{array}$ \\
\hline
\end{tabular}


Supplementary Table S3. Comparation of DeepCGSA performances with different network depth and sizes for SASA estimations of protein structures. R represents the Pearson correlation coefficient.

\begin{tabular}{|c|c|c|c|c|c|c|c|c|c|c|}
\hline \multirow{2}{*}{ 1D-ResNet } & \multirow{2}{*}{ 2D-ResNet } & \multicolumn{3}{|c|}{ C $\alpha$-based } & \multicolumn{3}{|c|}{$\mathrm{C} \alpha-\mathrm{C} \beta$} & \multicolumn{3}{|c|}{ Martini } \\
\hline & & $\begin{array}{l}\text { training set } \\
\text { loss }\end{array}$ & $\begin{array}{l}\text { validation set } \\
\text { loss }\end{array}$ & $\begin{array}{l}\text { validation set } \\
\text { residue-wise } R\end{array}$ & $\begin{array}{l}\text { training set } \\
\text { loss }\end{array}$ & $\begin{array}{l}\text { validation set } \\
\text { loss }\end{array}$ & $\begin{array}{l}\text { validation set } \\
\text { residue-wise } R\end{array}$ & $\begin{array}{l}\text { training set } \\
\text { loss }\end{array}$ & $\begin{array}{l}\text { validation set } \\
\text { loss }\end{array}$ & $\begin{array}{l}\text { validation set } \\
\text { residue-wise } R\end{array}$ \\
\hline $\begin{array}{c}2 \text { convolution layers ( } 16 \text { channels) } \\
4 \text { blocks with } 32 \text { channels } \\
5 \text { blocks with } 64 \text { channels }\end{array}$ & $\begin{array}{l}5 \text { blocks with } 16 \text { channels } \\
6 \text { blocks with } 32 \text { channels } \\
7 \text { blocks with } 64 \text { channels }\end{array}$ & 0.005009 & 0.005644 & 0.957 & 0.001026 & 0.001265 & 0.990 & 0.001502 & 0.001576 & 0.991 \\
\hline $\begin{array}{c}1 \text { convolution layer ( } 4 \text { channels) } \\
1 \text { convolution layer ( } 8 \text { channels) } \\
1 \text { block with } 8 \text { channels } \\
2 \text { blocks with } 16 \text { channels }\end{array}$ & $\begin{array}{l}2 \text { blocks with } 8 \text { channels } \\
3 \text { blocks with } 16 \text { channels }\end{array}$ & 0.005952 & 0.006122 & 0.953 & 0.002320 & 0.002354 & 0.983 & 0.001592 & 0.001624 & 0.990 \\
\hline $\begin{array}{l}1 \text { convolution layer ( } 4 \text { channels) } \\
2 \text { blocks with } 8 \text { channels }\end{array}$ & $\begin{array}{l}1 \text { block with } 8 \text { channels } \\
2 \text { blocks with } 16 \text { channels }\end{array}$ & 0.007057 & 0.007178 & 0.945 & 0.002690 & 0.002734 & 0.980 & 0.001927 & 0.001957 & 0.988 \\
\hline $\begin{array}{c}1 \text { convolution layer ( } 4 \text { channels) } \\
1 \text { block with } 8 \text { channels }\end{array}$ & 2 blocks with 8 channels & 0.008165 & 0.008320 & 0.936 & 0.006684 & 0.006756 & 0.945 & 0.003387 & 0.003414 & 0.978 \\
\hline
\end{tabular}


Supplementary Table S4. Comparation of DeepCGSA performances with different network depth and sizes for SASA estimations of RNA structures. R represents the Pearson correlation coefficient.

\begin{tabular}{|c|c|c|c|c|c|c|c|}
\hline \multirow{2}{*}{ 1D-ResNet } & \multirow{2}{*}{ 2D-ResNet } & \multicolumn{3}{|c|}{ P-based } & \multicolumn{3}{|c|}{ 3SPN } \\
\hline & & $\begin{array}{l}\text { training set } \\
\text { loss }\end{array}$ & $\begin{array}{l}\text { validation set } \\
\text { loss }\end{array}$ & $\begin{array}{l}\text { validation set } \\
\text { residue-wise } R\end{array}$ & $\begin{array}{l}\text { training set } \\
\text { loss }\end{array}$ & $\begin{array}{l}\text { validation set } \\
\text { loss }\end{array}$ & $\begin{array}{l}\text { validation set } \\
\text { residue-wise } R\end{array}$ \\
\hline $\begin{array}{c}2 \text { convolution layers ( } 16 \text { channels) } \\
4 \text { blocks with } 32 \text { channels } \\
5 \text { blocks with } 64 \text { channels }\end{array}$ & $\begin{array}{l}5 \text { blocks with } 16 \text { channels } \\
6 \text { blocks with } 32 \text { channels } \\
7 \text { blocks with } 64 \text { channels }\end{array}$ & 0.000235 & 0.009290 & 0.824 & 0.000105 & 0.005232 & 0.944 \\
\hline $\begin{array}{c}1 \text { convolution layer ( } 4 \text { channels) } \\
1 \text { block with } 8 \text { channels } \\
2 \text { blocks with } 16 \text { channels }\end{array}$ & $\begin{array}{l}2 \text { blocks with } 12 \text { channels } \\
3 \text { blocks with } 16 \text { channels }\end{array}$ & 0.007228 & 0.009015 & 0.827 & 0.003349 & 0.003731 & 0.961 \\
\hline $\begin{array}{l}1 \text { convolution layer ( } 4 \text { channels) } \\
2 \text { blocks with } 8 \text { channels }\end{array}$ & $\begin{array}{l}1 \text { block with } 8 \text { channels } \\
2 \text { blocks with } 16 \text { channels }\end{array}$ & 0.008135 & 0.008769 & 0.831 & 0.003834 & 0.004058 & 0.958 \\
\hline $\begin{array}{l}1 \text { convolution layer ( } 4 \text { channels) } \\
1 \text { block with } 8 \text { channels }\end{array}$ & 2 blocks with 8 channels & 0.013832 & 0.013889 & 0.718 & 0.017092 & 0.015373 & 0.759 \\
\hline
\end{tabular}


Supplementary Table S5. Comparations of DeepCGSA performance with different data reshaping operations in order to map the 1D-ResNet output tensor to 2D-ResNet input tensor for SASA estimations using C $\alpha$-based protein structures. R represents the Pearson correlation coefficient.

\begin{tabular}{cccc}
\hline Data reshaping operations & $\begin{array}{c}\text { training set } \\
\text { loss }\end{array}$ & $\begin{array}{c}\text { validation set } \\
\text { loss }\end{array}$ & $\begin{array}{c}\text { validation set } \\
\text { residue-wise R }\end{array}$ \\
\hline A) tensor duplication + B) transposition of duplicated tensor & 0.005952 & 0.006122 & 0.953 \\
A) tensor duplication & 0.006484 & 0.006634 & 0.949 \\
B) transposition of duplicated tensor & 0.007396 & 0.007525 & 0.942 \\
\hline
\end{tabular}

Supplementary Table S6. Comparisons of DeepCGSA performance with "mean" or "sum" operations to map the 2D-ResNet output tensor to the SASA values for SASA estimations using C $\alpha$-based protein structures. R represents the Pearson correlation coefficient.

\begin{tabular}{cccc}
\hline operations & $\begin{array}{c}\text { training set } \\
\text { loss }\end{array}$ & $\begin{array}{c}\text { validation set } \\
\text { loss }\end{array}$ & $\begin{array}{c}\text { validation set } \\
\text { residue-wise R }\end{array}$ \\
\hline "sum" & 0.005952 & 0.006122 & 0.953 \\
"mean" & 0.007706 & 0.007906 & 0.939 \\
\hline
\end{tabular}

\section{References}

(1) Hubbard, S. J.; Thornton, J. M., naccess. Computer Program, Department of Biochemistry and Molecular Biology, University College London $1993,2$.

(2) Kingma, D. P.; Ba, J., Adam: A method for stochastic optimization. 2014, arXiv:1412.6980.

(3) Onufriev, A. V.; Case, D. A., Generalized Born implicit solvent models for biomolecules. Annu. Rev. Biophys. 2019, 48, $275-296$.

(4) Honig, B.; Nicholls, A., Classical electrostatics in biology and chemistry. Science 1995, 268, 1144-1149.

(5) Sitkoff, D.; Sharp, K. A.; Honig, B., Accurate calculation of hydration free energies using macroscopic solvent models. J. Phys. Chem. 1994, 98, 1978-1988. 